University of Wollongong

Research Online

Faculty of Informatics - Papers (Archive)

Faculty of Engineering and Information

Sciences

2005

\title{
Measuring the linewidth enhancement factor of semiconductor lasers based on weak optical feedback effect
}

\author{
Yanguang Yu \\ University of Wollongong, yanguang@uow.edu.au \\ Jiangtao Xi \\ University of Wollongong, jiangtao@uow.edu.au \\ Enbang Li \\ University of Wollongong, enbang@uow.edu.au \\ Joe F. Chicharo \\ University of Wollongong, chicharo@uow.edu.au
}

Follow this and additional works at: https://ro.uow.edu.au/infopapers

Part of the Physical Sciences and Mathematics Commons

\section{Recommended Citation}

Yu, Yanguang; Xi, Jiangtao; Li, Enbang; and Chicharo, Joe F.: Measuring the linewidth enhancement factor of semiconductor lasers based on weak optical feedback effect 2005.

https://ro.uow.edu.au/infopapers/2874

Research Online is the open access institutional repository for the University of Wollongong. For further information contact the UOW Library: research-pubs@uow.edu.au 


\title{
Measuring the linewidth enhancement factor of semiconductor lasers based on weak optical feedback effect
}

\author{
Abstract \\ Semiconductor lasers are very different from other lasers because refraction variation can't be avoided \\ when the gain is changed. Refraction variation can be introduced the theory of semiconductor laser by a \\ dimensional parameter. This parameter is called linewidth enhancement factor (LEF). The value of LEF is \\ very important for many aspects of laser behavior. The LEF characterizes the linewidth broadening and \\ chirp due to fluctuation in the carrier density. A simple method to measure the linewidth enhancement \\ factor of laser diodes is presented in this paper. The method uses the self-mixing effect at a weak \\ feedback level. An optical beam is reflected and injected into the laser diode cavity by an external target, \\ and is then mixed with the light inside the cavity, causing variations of the optical output power. The \\ waveform of the optical power is determined by the feedback factor $\mathrm{C}$ and the LEF. A theoretical formula \\ to compute LEF is proposed for the case when the feedback level $C$ is smaller than 1 . The experimental \\ results show this method is feasible and simple when a laser diode operates at single longitudinal mode.

\section{Disciplines} \\ Physical Sciences and Mathematics

\section{Publication Details} \\ Yu, Y., Xi, J., Li, E. \& Chicharo, J. F. (2005). Measuring the linewidth enhancement factor of semiconductor \\ lasers based on weak optical feedback effect. In J. Yao, Y. Chen \& S. Lee (Eds.), Proceedings of SPIE \\ Volume: 5628: Semiconductor Lasers and Applicatiions II (pp. 34-39). USA: The Society of Photo-Optical \\ Instrumentation Engineers.
}




\title{
Measuring the Linewidth Enhancement Factor of Semiconductor Lasers Based on Weak Optical Feedback Effect
}

\author{
Yanguang $\mathrm{Yu}{ }^{*}{ }^{\mathrm{a}) b}$, Jiangtao $\mathrm{Xi}^{\text {a) }}$, Enbang $\mathrm{Li}^{\text {a)c }}$, Joe F. Chicharo ${ }^{\text {a) }}$ \\ ${ }^{a}$ School of Electrical, Computer and telecommunications Engineering \\ University of Wollongong, NSW 2522, Australia \\ ${ }^{\mathrm{b}}$ College of Information Engineering, Zhengzhou University, Zhengzhou, 450052 \\ 'Opto-electronics Information Science and Technology Laboratory, College of Precision Instrument \\ and Opto-Electronics Engineering, Tianjin University, P.R.China
}

\begin{abstract}
Semiconductor lasers are very different from other lasers because refraction variation can't be avoided when the gain is changed. Refraction variation can be introduced the theory of semiconductor laser by a dimensional parameter $\alpha$. This parameter is called linewidth enhancement factor (LEF). The value of LEF is very important for many aspects of laser behavior. The LEF characterizes the linewidth broadening and chirp due to fluctuation in the carrier density. A simple method to measure the linewidth enhancement factor of laser diodes is presented in this paper. The method uses the selfmixing effect at a weak feedback level. An optical beam is reflected and injected into the laser diode cavity by an external target, and is then mixed with the light inside the cavity, causing variations of the optical output power. The waveform of the optical power is determined by the feedback factor $\mathrm{C}$ and the LEF. A theoretical formula to compute LEF is proposed for the case when the feedback level $\mathrm{C}$ is smaller than 1 . The experimental results show this method is feasible when a laser diode operates at single longitudinal mode.
\end{abstract}

Keywords: Linewidth enhancement factor, optical feedback self-mixing interferometry, semiconductor laser

\section{INTRODUCTION}

Semiconductor lasers (SLs) are very different from other lasers because refraction variation can not be avoided when the gain is changed. Refraction variation can be introduced the theory of semiconductor laser by a dimensional parameter $\alpha$. This parameter is called linewidth enhancement factor (LEF). The value of $\alpha$ is very important for many aspects of laser behavior. It characterizes the linewidth broadening and chirp due to fluctuation in the carrier density.

Accurate measurement of $\alpha$ and $C$ is an important issue as they characterize the linewidth, the chirp, injection lock range, dynamic performances as well as all kinds of optical feedback effect in SLs. During the past decades people have done extensive research work on measuring $\alpha$ [1] and proposed many different approaches, such as the methods relying on the direct measurement of the relationship between sub-threshold optical spectrum and the injected current[2], the methods based on RF measurements[3] and the techniques based on the analysis of the locking regimes induced by optical injection from a master laser.[4,5] However, most existing approaches require sophisticated system setups or involve many complicated procedures, which thus make implementation difficult. With regard to the measurement of $C$, the task is more challenging and there is not much work reported yet.

The technique based on optical feedback self-mixing effect is an emerging technique in which a small fraction of the laser beam backscattered by a target is re-entering the laser active cavity. Initially, this back-reflection was seen as a major source of disturbance, strongly changing both frequency and amplitude of the lasing field. Presently, sensors based on this physical phenomenon in low-cost commercial laser diodes (LD) have been designed in a variety of applications such as dimensional control, vibration measurements, blood flow measurement, sound reproduction, angle measurements or even mass-market mobile telephones[6,7]. Applying this new technique on measuring $\alpha$ and $\mathrm{C}$ will be significant issue.

In 2004, an new approach [8] was proposed for the measuring $\alpha$ just based on the self-mixing optical feedback effect. However the approach can only be used for the case of a moderate feedback regime with $1<\mathrm{C}<3$. This paper, is also 
based on same principle, to measure $\alpha$ but for the weak feedback that is in the case of $0<\mathrm{C}<1$. Using this method, no strict requirement for the applied external cavity but in the approach [8] a symmetric return movement of the external cavity must be provided.

The paper is organized as follows: Section 2 briefly reviews the theory of self-mixing optical feedback interferometric effect, where a set of well-known mathematical models is presented and the formula used for calculating $\alpha$ and $\mathrm{C}$ are deduced. Then in Section 3 the experimental set-up and the measuring method are described and the testing results on $\alpha$ values for the different types laser diodes (LDs) are listed. Finally Section 4 concludes the paper.

\section{MEASUREMENT THEORY}

There are two alternative and equivalent methods for the analysis of self-mixing optical feedback inteferometric effects: the Long and Kobayashi equations based approach [9] and the three-mirror cavity based approach [10]. Both approaches yield the same description about the behavior of a single-mode SL with optical feedback, given by the following equations:

$$
\begin{aligned}
& \phi_{F}(\tau)=\phi_{0}(\tau)-C \cdot \sin \left[\phi_{F}(\tau)+k\right] \\
& \left.P\left(\phi_{F}(\tau)\right)=P_{0} \mid 1+m G\left(\phi_{F}(\tau)\right)\right] \\
& G\left(\phi_{F}(\tau)\right)=\cos \left(\phi_{F}(\tau)\right)
\end{aligned}
$$

Equation (1) is called the phase equation, equation (2) describes laser emitting power or self-mixing signal, and equation (3) is interference function. Where $k=\arctan (\alpha)$ and $\alpha$ is linewidth enhancement factor; $\phi_{0}(\tau)=\omega_{0} \tau$ and $\phi_{F}(\tau)=\omega_{F}(\tau) \tau$, where $\omega_{0}$ and $\omega_{F}(\tau)$ are the angular frequencies of the SL without and with feedback respectively; $\tau=2 L / c$, where $L$ is the length of the external cavity and $c$ the speed of light; $C$ is the feedback level factor.

The above parameters are described in more details as follows: $\alpha$ is defined as $\alpha=\frac{\partial n_{R} / \partial N}{\partial n_{I} / \partial N}$, where $N, n_{R}, n_{I}$ are the carrier density in laser medium, the real and imaginary part of the refractive index respectively. $C=\varepsilon \frac{L \cdot \sqrt{1+\alpha 2}}{l \cdot n} \sqrt{R_{\text {ext }}} \frac{1-R_{2}}{\sqrt{R_{2}}}$, where $R_{2}$ is the power reflectivity of the SL output facet; $R_{\text {ext }}$ is the reflectivity of the external target; $l$ is SL cavity length; $n$ is SL cavity refractive index and $\varepsilon$ is an coefficient that accounts for spatial mode overlap mismatch between the back-reflected light and the lasing mode (typically $\varepsilon=0.1-0.8$ ).

In Equation (2), $P\left(\phi_{F}(\tau)\right)$ and $P_{0}$ are the power emitted by the SL with and without the external cavity respectively. It is seen that with the external cavity, the emitted power deviated from $P_{0}$ by a factor of $m G\left(\phi_{F}(\tau)\right)$ where $m$ is called modulation index (typical $m \approx 10^{-3}$ ), and interferometric function $G\left(\phi_{F}(\tau)\right)$ gives the effect of the external light phase to the emitted power.

With a self-mixing experimental setup, a self-mixing signal $P\left(\phi_{F}(\tau)\right)$ can be detected. Clearly $G\left(\phi_{F}(\tau)\right)$ can be obtained from $P\left(\phi_{F}(\tau)\right)$. We will give a theoretical formula for calculating LEF by analysizing $G\left(\phi_{F}(\tau)\right)$. When $\mathrm{C}<1$, the simulation waveform of $G\left(\phi_{F}(\tau)\right)$ is shown as in figure 1 . 


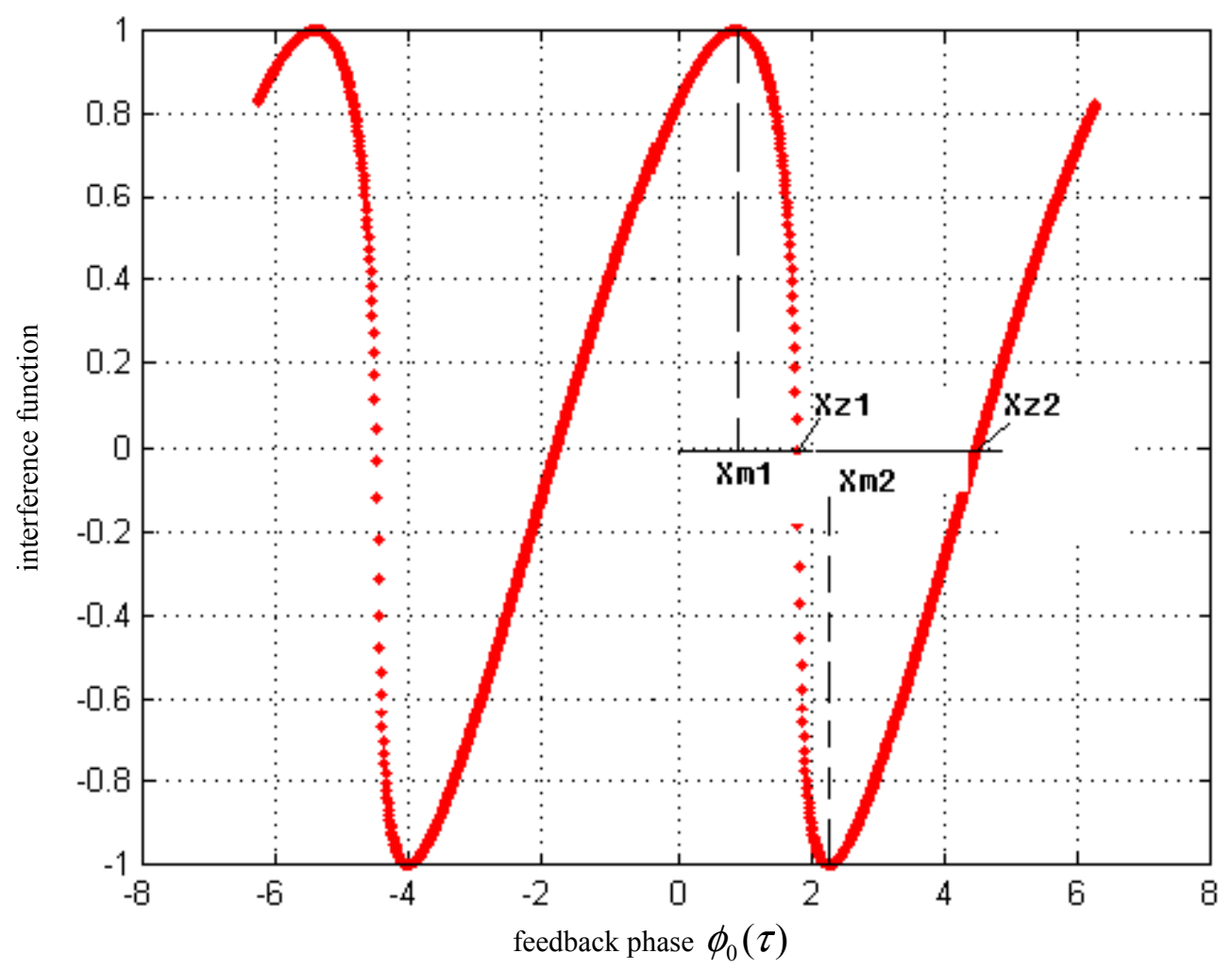

Figure 1 the waveform of $G\left(\phi_{F}(\tau)\right)$ with parameters of $\mathrm{C}=0.9$ and $\alpha=4$

From equations (1) and (3), we have

$$
\partial G\left(\phi_{F}(\tau)\right) / \partial \phi_{0}(\tau)=-\frac{\sin \left(\phi_{F}(\tau)\right)}{1+C \cos \left(\phi_{F}(\tau)+\arctan (\alpha)\right)}
$$

in the range of $[0,2 \pi]$, let $\partial G\left(\phi_{F}(\tau)\right) / \partial \phi_{0}(\tau)=0$, the abscissa values of the two peaks marked as $\mathrm{x}_{\mathrm{M} 1} \mathrm{x}_{\mathrm{M} 2}$ on figure 1 can be expressed as

$$
\begin{aligned}
& x_{M 1}=C \sin (\arctan (\alpha)) \\
& x_{M 2}=\pi-C \sin (\arctan (\alpha))
\end{aligned}
$$

the abscissa values of the zero-crossing points marked as $\mathrm{x}_{\mathrm{Z} 1} \mathrm{x}_{\mathrm{Z} 2}$ on fig. 1 are

$$
\begin{aligned}
& x_{Z 1}=\frac{\pi}{2}+C \times \cos (\arctan (\alpha)) \\
& x_{Z 2}=\frac{3 \pi}{2}-C \times \cos (\arctan (\alpha))
\end{aligned}
$$

from equations (5)-(8), we can get

$$
\begin{aligned}
& k_{1}=\left(x_{\mathrm{M} 2}-x_{M 1}\right) / 2 \pi=0.5-C \sin (\arctan (\alpha)) / \pi \\
& k_{2}=\left(x_{Z 2}-x_{Z 1}\right) / 2 \pi=0.5-C \cos (\arctan (\alpha)) / \pi
\end{aligned}
$$

by equation (9) and (10)

$$
\alpha=\left|\frac{0.5-k_{1}}{0.5-k_{2}}\right|
$$

We can determine factors $\alpha$ by measuring $\mathrm{k} 1$ and $\mathrm{k} 2$. The Equation (11) is the basic formula used to measure $\alpha$. Then, by using of the measured $\alpha$, from equation (9) or (10), we may calculate $\mathrm{C}$ value. 


\section{EXPERIMENTAL RESULTS}

A block diagram of the experimental setup for measuring $\alpha$ based on self-mixing effects is shown in Fig. 2 . The system comprises the laser diode biased with a dc injection current far above its threshold, the collimating microscope objective, and an external reflecting target (PZT or loudspeaker). The target, driven by a triangular or sinusoid voltage, modulates the feedback optical phase in the LD and produces variation in the laser power. The laser beam emitted from the LD's rear facet is monitored by a photodiode (PD) within the LD package. The signal from the PD is detected by a processing circuit and then recorded by a digital oscilloscope.

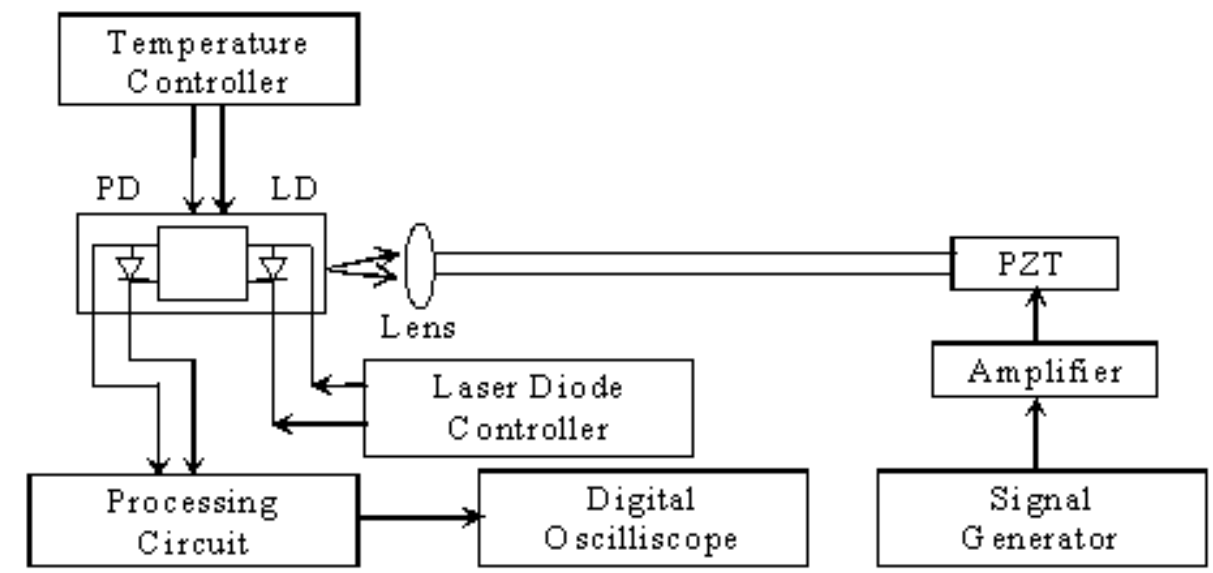

Figure 2 Experimental setup for measuring $\alpha$ based on optical feedback self-mixing effect

A typical self-mixing signal is obtained from the above experimental setup and shown in figure 3 , with some
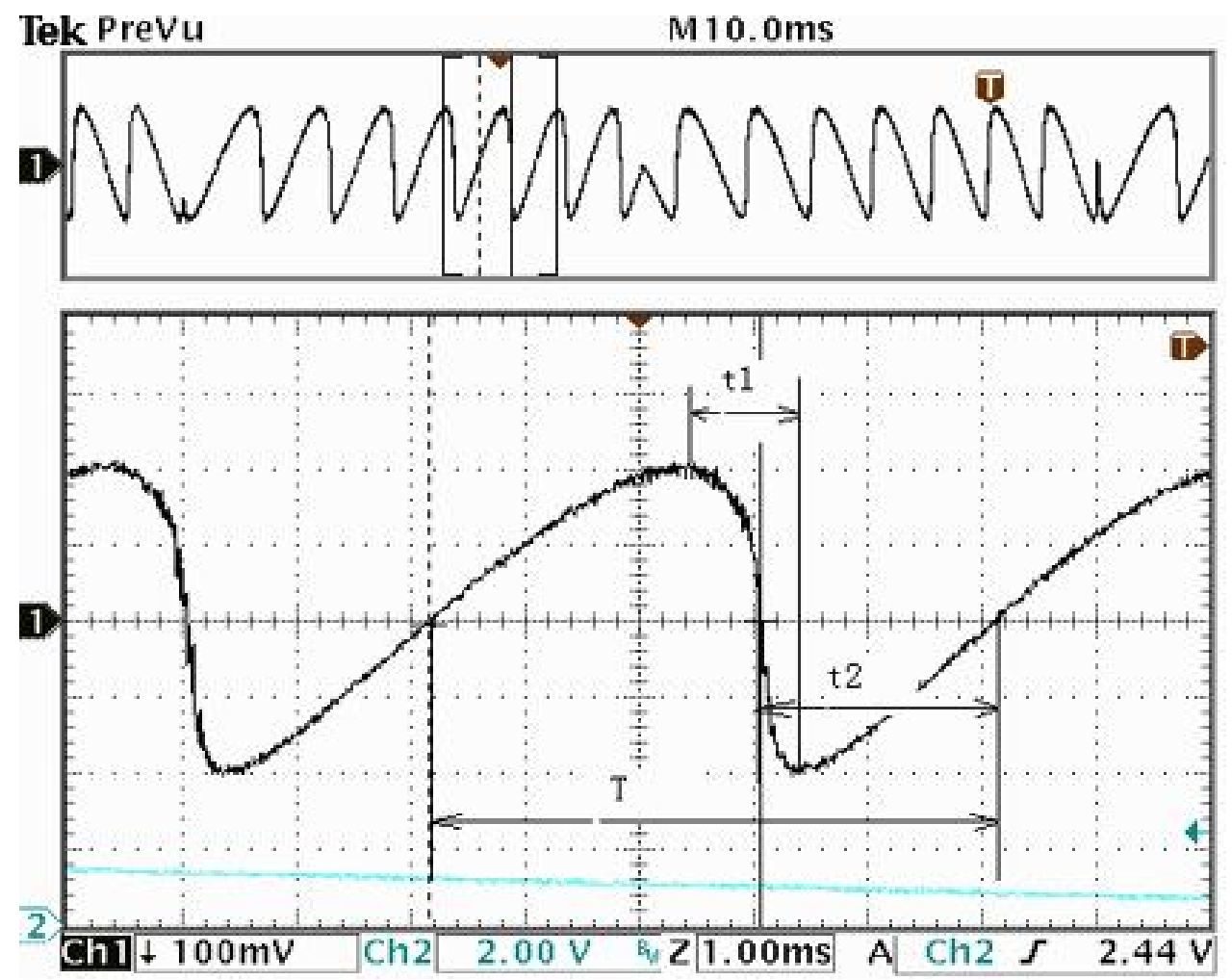

Figure 3 Experimental self-mixing signal 
measurement parameters marked on it. We can get $\mathrm{k} 1, \mathrm{k} 2$ by the following measuring equations:

$$
\begin{aligned}
& k_{1}=\frac{t_{1}}{T} \\
& k_{2}=\frac{t_{2}}{T}
\end{aligned}
$$

The values of $t_{1}$ and $t_{2}$ and $T$ are defined in figure 3 .

We tested $\alpha$ values for 3 different types laser diodes by the above method, the experimental results are listed in the table 1. These results are agreed with the values reported in reference[ 8].

Table 1. Experimental results

\begin{tabular}{|c|c|c|c|c|}
\hline Type & from & descriptions & $\alpha$ & error \\
\hline SDL-7511-G1 & SDL & MQW,635nm, DFB & 2.2 & $\pm 8.5 \%$ \\
\hline DL7140-201 & SANYO & AlGaInP,785nm, MQW & 3.0 & $\pm 6.9 \%$ \\
\hline HL8325G & HITACH & GaAlAs,820nm, TQW & 3.3 & $\pm 5.5 \%$ \\
\hline
\end{tabular}

Test considition: feedback level $\mathrm{C}$ is smaller than 1 ; temperature controller at $25 \pm 0.1^{\circ} \mathrm{C}$; Injection current is far above the threshold; LDs operate at single mode. DFB: Distributed Feedback, MQW: Multiple Quantum Well, TQW: Triple Quantum Well; error is calculated as standard deviation of the measured data.

There are 3 main sources of error existed in this experimental setup. One is the low frequency fluctuation from the detecting circuit, which brings out error to measure zero base of the self-mixing signal. One is mode hopping in the laser diode or high optical noise, which can change the waveform of the self-mixing signal. Another one is due to the ambiguity of the peak positions on the waveform. In our experiment, the mode hopping is avoided by controlling the temperature and limiting the maximum feedback level. The low frequency fluctuation effect can be reduced by using high pass filter if applying a higher driving frequency on the target. The accurate identification for the peak positions may be achieved by data processing and fitting. In contrast to approach [8], the propose method in this paper doesn't require strictly symmetry for the return movement of the external target.

\section{CONCLUSION}

We have presented a new approach to estimate the linewidth enhancement factor of SLs. This method is based on the self-mixing optical feedback effects, in which the SL operates at weak optical feedback regime with $\mathrm{C}<1$. The effectiveness for the proposed method has been confirmed from the experiments.

\section{ACKNOLEDGEMENT}

This work was partly supported by the Scientific Research Foundation of Henan Province (042420178).

\section{REFERENCES}

1. M. Osinski, and J. Buus, "Linewidth broadening factor in semiconductor lasers - an overview", IEEE J. Quantum Electron., vol. 23, pp. 9-28, 1987.

2. Piotr Konrad Kondratko, Shun_lien Chuang, Gabriel Walter, Theodore Chung, and Nick Holonyak Jr., "Observations of near-zero linewidth enhancemenr factor in a quantum-well coupled quantum-dot laser", Applied physics letters, vol. 83(23), pp. 4818-4820, 2003

3. K. Kikuchi, T Okoshi, "Estimation of linewidth enhancement factor of AlGaAs lasers by correlation measurement between FM and AM noises", IEEE J. Quantum Electron., vol. 21, no. 6, pp. 669-673, 1985

4. G. Liu, X. Jin, and S.L. Chuang, "Measurement of linewidth enhancement factor of semiconductor lasers using an injection-locking technique", IEEE Photon. Technol. Lett., vol. 13, pp. 430-432, 2001.

5. K.E. Chlouverakis, K.M. Al-Aswad, I.D. Henning, M.J Adams, "Determining laser linewidth parameter from Hopf bifurcation minimum in lasers subject to optical injection" Electron. Lett., vol. 39, pp. 1185 -1187, 2003. 
6. G. Giuliani, M. Norgia, S. Donati, T. Bosch, “ Laser diode self-mixing technique for sensing applications”, J. Opt. A: Pure Appl. Opt., 4(6): S283-S294, 2002

7. Lorenzo Scalise, Yanguang Yu, Guido Giuliani, Guy Plantier and Thierry Bosch, “ Self-Mixing Laser Diode Velocimetry: Application To Vibration And Velocity Measurement", IEEE Transactions on Instrumentation and Measurement, vol. 53, n. 1, pp. 223-232, 2004.

8. Yanguang Yu, Guido Giuliani, Silvano Donati. "Measurement of the Linewidth Enhancement Factor of Semiconductor Lasers based on the Optical Feedback Self-Mixing Effect", IEEE Photon. Technol. Lett. 16(4):990992, 2004

9. S. Donati, G. Giuliani, and S.Merlo, "laser diode feedback interferometer for measurement of displacements without ambiguity”, IEEE J. Quantum Electron. 31(1), 113-119, 1995

10. G. Mourat, N. Servagent, and T. Bosch, "optical feedback effects on the spectral linewidth of semiconductor lasers using the self-mixing interference ”, IEEE J. Quantum Electron. 34(9), 1717-1721, 1998

yanguangyu@,zzu.edu.cn; yanguang@uow.edu.au; office phone 86371 7763882-2112 